\title{
Magnetic main sequence stars as progenitors of blue supergiants
}

\author{
I. Petermann, N. Castro and N. Langer \\ Argelander Institute for Astronomy, Bonn, Germany \\ email: ilka@astro.uni-bonn.de
}

\begin{abstract}
Blue supergiants (BSGs) to the right the main sequence band in the HR diagram can not be reproduced by standard stellar evolution calculations. We investigate whether a reduced convective core mass due to strong internal magnetic fields during the main sequence might be able to recover this population of stars. We perform calculations with a reduced mass of the hydrogen burning convective core of stars in the mass range $3-30 M_{\odot}$ in a parametric way, which indeed lead to BSGs. It is expected that these BSGs would still show large scale magnetic fields in the order of $10 \mathrm{G}$.
\end{abstract}

Keywords. Stars: massive - Stars: supergiants - Stars: magnetic field - Stars: evolution

\section{Introduction and setup of calculations}

Magnetic massive stars are rare, until recently only three magnetic O-type stars were known (Donati et al. 2002, 2006; Bouret et al. 2008). However, recently new observations have increased this number significantly (Grunhut et al. 2009; Hubrig et al. 2011; Fossati et al. 2014) and current estimates assume that about ten percent of OB-type stars host a magnetic field. Stable, large-scale magnetic fields are suggested to be of fossil origin, arising from fields in a molecular cloud that are conserved during star formation (Braithwaite \& Nordlund 2006). Alternatively, they can be explained as a result of the mergers of two main sequence stars. Binary interaction resets the evolutionary clock of the star. Therefore we assumed that the B-field acts unaltered since the ZAMS of the rejuvenated star (de Mink et al. 2014). Many observed magnetic field, mostly of dipole characteristics, are expected to be stabilized by an internal toroidal field (Braithwaite \& Spruit 2004). This field is assumed to interact and compress the core convection, resulting in a core with reduced size. Zahn (2009) have shown that even moderate magnetic fields can already induce significant effects. For this work, the stellar evolution code BEC was used, see for example Köhler et al. (2012) for details. We simulate a reduced convective core as a result of an interaction with a magnetic field by applying a modified Ledoux criterion

$$
\nabla_{\mathrm{rad}}<f * \nabla_{\text {Led }},
$$

with

$$
\nabla_{\text {Led }}=\nabla_{\text {ad }}-\frac{\chi_{\mu}}{\chi_{T}} \nabla_{\mu}
$$

and chose values for a "reduction parameter" $f$ between 1.12 and 1.20. These values are shown to reduce the mass of the convective core sufficiently for the current purpose, although higher values might be necessary for stars with masses lower than $3 M_{\odot}$. 


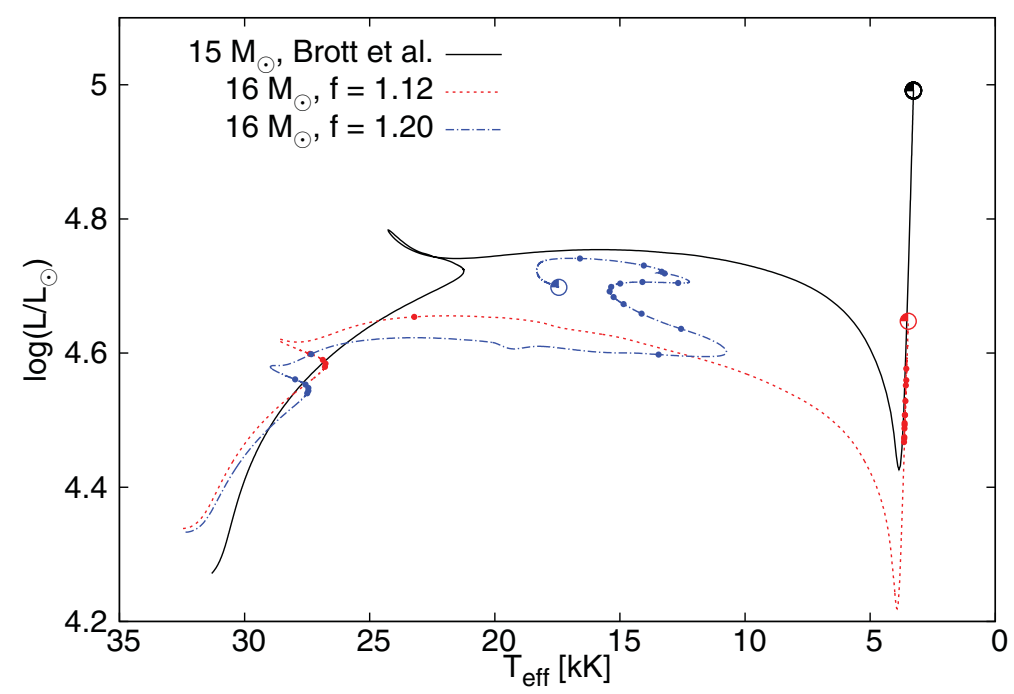

Figure 1. Evolutionary track of a $16 M_{\odot}$ star in the HR diagram, computed with two different reduction parameters. For the smaller value, $\mathrm{f}=1.12$, the star continues its evolution as a red supergiant (dashed line), however its final luminosity is significantly smaller than in standard calculations - see for a comparison the track for a $15 M_{\odot}$ star (solid line) by Brott et al. (2011). For the larger value, $f=1.20$, the red supergiant stage is avoided (dot-dashed line). The dots indicate timesteps of $10^{5}$ years.

\section{Results and discussion}

We showed that with a sufficiently reduced convective core, magnetic stars no longer evolve as red supergiants, but remain in the blue part of the HR diagram, thus filling the post-main sequence gap that remains otherwise unpopulated. Those stars that retain a convective core that is still large enough for an evolution as a red supergiant, show a final luminosity that is smaller when compared to standard calculations (see Fig. 1). In general, a trend is seen that for smaller masses, a larger reduction parameter is required for avoiding the red supergiant stage. Below $8 M_{\odot}$ the red supergiant stage is avoided, even for the largest values of $f$. With the assumption that stars on the main sequence have surface magnetic fields of the order of $1 \mathrm{kG}$, those of magnetic blue supergiants are expected to have values around $10 \mathrm{G}$ and are therefore likely observable.

\section{References}

Bouret, J.-C., Donati, J.-F., Martins, F., et al. 2008, MNRAS 389, 75

Braithwaite, J. \& Nordlund, A. 2006, A\&A 450, 1077

Braithwaite, J. \& Spruit, H. C. 2004, Nature 431, 819

Brott, I., de Mink, S. E., Cantiello, M., et al. 2011, A\&A 530, A115

de Mink, S. E., Sana, H., Langer, N., Izzard, R. G., \& Schneider, F. R. N. 2014, ApJ 782, 7

Donati, J.-F., Babel, J., Harries, T. J., et al. 2002, MNRAS 333, 55

Donati, J.-F., Howarth, I. D., Bouret, J.-C., et al. 2006, MNRAS 365, L6

Fossati, L., Zwintz, K., Castro, N., et al. 2014, A\&A 562, A143

Grunhut, J. H., Wade, G. A., Marcolino, W. L. F., et al. 2009, MNRAS 400, L94

Hubrig, S., Schöller, M., Kharchenko, N. V., et al. 2011, A\& A 528, A151

Köhler, K., Borzyszkowski, M., Brott, I., Langer, N., \& de Koter, A. 2012, A 8 A 544, A76

Zahn, J.-P. 2009, Communications in Asteroseismology 158, 27 